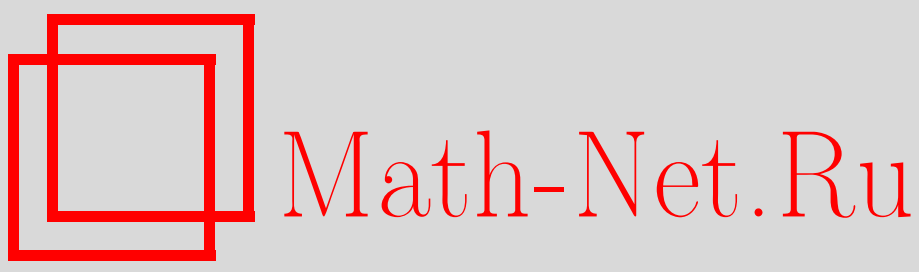

Д. Н. Иванов, Аналог теоремы Вагнера о разложениях алгебры матриц, Матем. сб., 2004, том 195, номер 11, 13-30

DOI: https://doi.org/10.4213/sm857

Использование Общероссийского математического портала Math-Net.Ru подразумевает, что вы прочитали и согласны с пользовательским соглашением http://www . mathnet.ru/rus/agreement

Параметры загрузки:

IP : 44.207 .124 .84

26 апреля 2023 г., 17:51:07 
УДК 512.55

\author{
Д. Н. Иванов
}

\title{
Аналог теоремы Вагнера о разложениях алгебры матриц
}

\begin{abstract}
Знаменитая теорема Вагнера утверждает, что конечная аффинная плоскость, обладающая 2-транзитивной на прямых группой коллинеаций, является плоскостью трансляций. Понятие ортогонального разложения (OP) классически полупростой ассоциативной алгебры, введенное автором, позволяет провести аналогию между конечными афффинньми плоскостями порядка $n$ и OP алгебры матриц $\mathrm{M}_{n}(\mathbb{C})$ в сумму подалгебр, сопряженных диагональной. Эти ОР назьваются WP-разложениями и эквивалентны известному понятию OP простых алгебр Ли типа $A_{n-1}$ в сумму картановских подалгебр. В статье приводится подробное и улучшенное доказательство аналога теоремы Вагнера для WP-разложений алгебры матриц нечетного неквадратного порядка, схема которого была ранее опубликована в краткой заметке в журнале "Успехи математических наук" в 1994 г. Кроме того, в рамках теории ОР ассоциативных алгебр на основе метода идемпотентных базисов получено элементарное доказательство известной теоремы Кострикина-Тьепа о неприводимых ОР алгебры Ли типа $A_{n-1}$ в случае примарного $n$.
\end{abstract}

Библиография: 18 названий.

\section{§1. Введение}

Знаменитая теорема Вагнера [1], [2] утверждает, что конечная аффинная плоскость, обладаюшая 2-транзитивной на прямых группой коллинеаций, является плоскостью трансляций. Понятие ортогонального разложения (OP) классически полупростой ассоциативной алгебры, введенное автором в [3], позволяет провести аналогию между конечными аффинными плоскостями порядка $n$ и ОР алгебры матриц $\mathrm{M}_{n}(\mathbb{C})$ в сумму подалгебр, сопряженных диагональной. Эти ОР называются WP-разложениями и эквивалентны известному понятию ОР простых алгебр Ли типа $A_{n-1}$ в сумму картановских подалгебр [4]. В статье приводится подробное и улучшенное доказательство аналога теоремы Вагнера для WP-разложений алгебры матриц нечетного неквадратного порядка, схема которого была ранее опубликована в [5]. Кроме того, в рамках теории ОР ассоциативных алгебр на основе метода идемпотентых базисов получено элементарное доказательство известной теоремы Кострикина-Тьепа о неприводимых ОР алгебры Ли типа $A_{n-1}$ в случае примарного $n$.

Напомним основные определения. Пусть $\mathbf{A}$ - ассоциативная конечномерная над полем комплексных чисел $\mathbb{C}$ алгебра, через $\operatorname{Tr}_{A}$ обозначим следправого регулярного представления $\mathbf{A}$. Тогда $\operatorname{Tr}_{A} x y$ является симметричной инвариантной формой

Работа вьполнена при поддержке Российского фонда фундаментальных исследований (грант № 02-01-00219).

(С) Д.Н. Иванов 2004 
на $\mathbf{A}$, которую мы будем называть формой следа. Известно, что алгебра $\mathbf{A}$ полупроста тогда и только тогда, когда форма следа невырождена на А. Далее термин "алгебра", если не оговорено противное, будет обозначать ассоциативную конечномерную над $\mathbb{C}$ полупростую алгебру. Таким образом, по теореме Веддербарна алгебра $\mathbf{A}$ изоморфна конечной прямой сумме матричных алгебр $\mathrm{M}_{n}(\mathbb{C})=\mathrm{M}_{n}$. Такие алгебры назьвают также классически полупростыми.

ОПРЕДЕЛЕнИЕ. Семейство неединичных собственных подалгебр $\mathscr{D}=\left\{\mathbf{B}_{i}\right.$, $i=1, \ldots, r\}$ образует ортогональное разложение $(\mathrm{OP})$ алгебры $\mathbf{A}$, если

(1) все подалгебры $\mathbf{B}_{i}$ полупросты и содержат единичный элемент $\mathbf{1}_{A}$ алгебры $\mathbf{A}$;

(2) (условие ортогональности) алгебра $\mathbf{A}$ является прямой суммой попарно ортогональных подпространств

$$
\mathbf{A}=\left\langle\mathbf{1}_{A}\right\rangle \oplus \mathbf{B}_{1}^{\circ} \oplus \cdots \oplus \mathbf{B}_{r}^{\circ}
$$

где $\mathbf{B}_{i}^{\circ}=\left\{x \in \mathbf{B}_{i} \mid \operatorname{Tr}_{A} x=0\right\}$.

Если все подалгебры ОР $\mathscr{D}$ коммутативны, то $\mathscr{D}$ называется коммутативныл. Если все подалгебры в $\mathscr{D}$ изоморфны алгебре $\mathbf{B}$, то $\mathscr{D}$ называется однородныц $O P$ mипа $\mathbf{B}$ (или просто OP типа $\mathbf{B}$ ).

Введем обозначения:

- через $k \mathrm{M}_{n}$ будем обозначать прямую сумму $k$ экземпляров алгебры матриц $\mathrm{M}_{n}$;

$-\operatorname{Pr}\left(\mathbf{B}_{i}\right)$ обозначает множество примитивных идемпотентов ${ }^{1}$ подалгебры $\mathbf{B}_{i}$,

$$
\operatorname{Pr}(\mathscr{D})=\operatorname{Pr}\left(\mathbf{B}_{1}\right) \cup \cdots \cup \operatorname{Pr}\left(\mathbf{B}_{n+1}\right)
$$

Однородное $\mathrm{OP}$ типа $n \mathrm{M}_{1}$ алгебры матриц $\mathrm{M}_{n}$ назьвается WP-разложением ("WP" происходит от "Winnie-the Pooh"). Изучение WP-разложений было начато А.И. Кострикиным, И.А. Кострикиным и В.А. Уфнаровским в рамках теории алгебр Ли как ортогональных разложений простых алгебр Ли типа $A_{n-1}$ в сумму картановских подалгебр [4]. Связь между двумя понятиями устанавливается следующим образом. Рассмотрим реализацию простой алгебры Ли $L$ типа $A_{n-1}$ в виде $(n \times n)$-матриц с нулевым следом. Пусть $L=H_{1} \oplus \cdots \oplus H_{n+1}-$ ортогональная прямая сумма картановских подалгебр. Тогда ассоциативные подалгебры $\left\{\mathbf{B}_{i}=\left\langle H_{i}, E_{n}\right\rangle_{\mathbb{C}}, i=1, \ldots, n+1\right\}$ образуют WP-разложение алгебры матриц $\mathrm{M}_{n}$ $\left(E_{n}\right.$ - единичная матрица порядка $\left.n\right)$.

Идея рассматривать WP-разложения как конфигурации идемпотентов в контексте понятия коммутативных ОР принадлежит автору. Эта идея позволила связать WP-разложения с теорией конечных афффинных плоскостей. Напомним, см. [3], что аффинная плоскость порядка $n$ соответствует однородному ОР типа $n \mathrm{M}_{1}$ алгебры $n^{2} \mathrm{M}_{1}$. Так что по существу разница между двумя понятиями заключается

\footnotetext{
${ }^{1}$ Идемпотент $e$ называется примитивньм, если не существует ненулевых идемпотентов $e_{1}, e_{2}$ таких, что $e=e_{1}+e_{2}$ и $e_{1} e_{2}=e_{2} e_{1}=0$.
} 
только в замене простой алгебры $\mathrm{M}_{n}$ на коммутативную алгебру $n^{2} \mathrm{M}_{1}$ той же самой размерности. Как будет видно далее, такой подход оказался весьма продуктивным.

Соответствие между аффинными плоскостями порядка $n$ и однородньми ОР типа $n \mathrm{M}_{1}$ алгебры $n^{2} \mathrm{M}_{1}$ устанавливается следующим образом: идемпотенты $\operatorname{Pr}\left(n^{2} \mathrm{M}_{1}\right)$ рассматриваются как точки, а идемпотенты $\operatorname{Pr}(\mathscr{D})$ как прямые, где $\mathscr{D}$ - OP типа $n \mathrm{M}_{1}$. В этой ситуации подалгебрам из $\mathscr{D}$ соответствуют классы параллельности.

Теперь мы можем переформулировать теорему Вагнера в терминах теории ОР. Напомним, что әлементарной абелевой группой ( $p$-группой) называется группа экспоненты $p$, где $p$ простое.

TеОрема ВаГнеРА. Пусть $\mathscr{D}$ - однородное OP muna $n \mathrm{M}_{1}$ алгебры $n^{2} \mathrm{M}_{1}$. Eсли Aut $\mathscr{D}$ действует транзитивно на $\operatorname{Pr}(\mathscr{D})$, то Aut $\mathscr{D}$ содержит нормальную әлементарную абелеву подгруппу порядка $n^{2}$, регулярную на $\operatorname{Pr}\left(n^{2} \mathrm{M}_{1}\right)$ и тождественную на $\mathscr{D}$ (другими словами, $\mathscr{D}$ соответствует плоскости транслячий).

Аналогом плоскостей трансляций среди WP-разложений служат так называемые Ј-разложения, которые конструируются из неприводимых представлений экстраспециальных групп. В рамках теории ОР ассоциативных алгебр мы можем сформулировать аналог теоремы Вагнера для WP-разложений.

ГипотезА 1 (аналог теоремы Вагнера). Если WP-разложение $\mathscr{D}$ алгебры $\mathrm{M}_{n}$ допускает группу автоморфизмов, транзитивную на $\operatorname{Pr}(\mathscr{D})$, то $\mathscr{D}$ сопряжено J-разложению $и$, в частности, Aut $\mathscr{D}$ содержит неприводимую нормальную әлементарную абелеву подгруппу порядка $n^{2}=p^{2 m}, \quad$ простое, действующую тождественно на $\mathscr{D}$.

Гипотеза 1 будет доказана далее для нечетных $n$, не являюшихся квадратами.

В направлении гипотезы 1 есть важный результат, установленный А. И. Кострикиньм и Фам Хыу Тьепом в [6] в рамках теории алгебр Ли. Мы переформулируем его в терминах ассоциативных алгебр:

$(*)$ Пусть $\mathscr{D}$ - WP-разложение алгебры $\mathrm{M}_{n}$. Если Aut $\mathscr{D}$ действует неприводимо на пространстве матриц с нулевым следом, то $\mathscr{D}$ сопряжено Ј-разложению.

WP-разложения, которые рассматриваются в $(*)$, называются неприводимыми

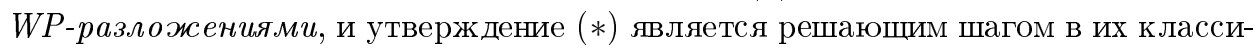
фикации. Проблема переводится в рамки теории плоскостей трансляций - остается только классифицировать симплектические расслоения с группой коллинеаций, транзитивной на ненулевых векторах. Это уже не так сложно сделать, учитьвая результаты Хупперта и Геринга о транзитивных линейных группах. Классификация неприводимых WP-разложений была начата в [7] и закончена в [6]. Это один из центральных результатов о WP-разложениях. Его подробное изложение и много другой информации о WP-разложениях и ортогональных разложениях простых алгебр Ли можно найти в книге [8].

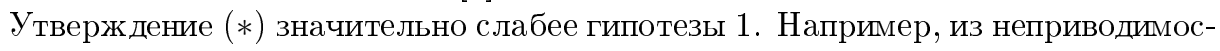
ти Aut $\mathscr{D}$ на пространстве матриц со следом нуль немедленно вытекает 2-транзитивность действия нормализатора в Aut $\mathscr{D}$ подалгебры $\mathbf{B}_{i} \in \mathscr{D}$ на множестве ее 
примитивных идемпотентов $\operatorname{Pr}\left(\mathbf{B}_{i}\right)$. Тогда как транзитивность Aut $\mathscr{D}$ на $\operatorname{Pr}(\mathscr{D})$ влечет лишш транзитивность нормализатора $\mathbf{B}_{i}$ на $\operatorname{Pr}\left(\mathbf{B}_{i}\right)$.

Доказательство утверждения $(*)$, приведенное в [6], сушественно использует классификацию 2-транзитивных групा и, следовательно, классификацию конечных простых групп. В заключительном разделе статьи приводится "элементарное" доказательство (*) для примарных $n$, не используюшее классификацию 2-транзитивных групп.

Отметим, что кроме аналога теоремы Вагнера в теории WP-разложений остается открытой известная проблема ( "проблема Винни-Пуха" [4]) о сушествовании WP-разложений в алгебрах матриц непримарного порядка. Более того, пока неизвестны примеры WP-разложений, не сопряженных J-разложениям.

Также отметим, что WP-разложения интенсивно изучаются. Им посвящена значительная часть монографии [8], где изложение ведется в терминах алгебр Ли. Связи WP-разложений с градуировками на алгебре матриц исследуются в [9]. $\mathrm{B}[10]$ установлено соответствие между WP-разложениями алгебры матриц $\mathrm{M}_{n}$ и сбалансированньми базисами алгебры $(n+1) \mathrm{M}_{1} \oplus \mathrm{M}_{n}$.

\section{§2. J-разложения и симплектические расслоения}

Напомним конструкцию так называемых Ј-разложений алгебры матриц $\mathrm{M}_{p^{n}}$. Определим $(p \times p)$-матрицы $D, P$ и $J_{a, b}$ :

$$
D=\left(\begin{array}{ccccc}
1 & & & \\
& \varepsilon & & & \\
& \varepsilon^{2} & & \\
& & \ddots & \\
& & & \varepsilon^{p-1}
\end{array}\right), \quad P=\left(\begin{array}{ccccc}
0 & 0 & \ldots & 0 & 1 \\
1 & 0 & \ldots & 0 & 0 \\
0 & 1 & \ldots & 0 & 0 \\
\ldots & \ldots \ldots & \ldots & \ldots \\
0 & 0 & \ldots & 0 & 1
\end{array}\right), \quad J_{a, b}=D^{a} P^{b}
$$

где $\varepsilon$ - примитивный корень степени $p$ из 1. Удобно рассматривать $a, b$ как элементы поля вычетов $\mathbb{F}_{p}=\mathbb{Z} / p \mathbb{Z}$. С каждым вектором $u=\left(a_{1}, \ldots, a_{n} ; b_{1}, \ldots, b_{n}\right) \in$ $V=\mathbb{F}_{p}^{2 n}$ свяжем матрицу

$$
J_{u}=J_{a_{1}, b_{1}} \otimes \cdots \otimes J_{a_{n}, b_{n}}
$$

Так как $D^{a} P^{b}=\varepsilon^{a b} P^{b} D^{a}$, то

$$
J_{u} J_{u^{\prime}} J_{u}^{-1}=\varepsilon^{\left\langle u \mid u^{\prime}\right\rangle} J_{u^{\prime}},
$$

где для $u=\left(a_{1}, \ldots, a_{n} ; b_{1}, \ldots, b_{n}\right)$ и $u^{\prime}=\left(a_{1}^{\prime}, \ldots, a_{n}^{\prime} ; b_{1}^{\prime}, \ldots, b_{n}^{\prime}\right)\left\langle u \mid u^{\prime}\right\rangle=$ $\sum_{i=1}^{n}\left(a_{i} b_{i}^{\prime}-a_{i}^{\prime} b_{i}\right)$ определяет невырожденную кососимметрическую билинейную форму на $V$.

WP-разложение $\mathscr{D}=\left\{\mathbf{B}_{1}, \ldots, \mathbf{B}_{p^{n}+1}\right\}$ назьвается $J$-разложением, если каждая подалгебра $B_{i}$ содержит базис из некоторых матриц $J_{u}$. В этом случае положим $V_{i}=\left\{u \mid J_{u} \in \mathbf{B}_{i}\right\}$. Тогда

(1) каждое $V_{i}$ является $\mathbb{F}_{p}$-подпространством размерности $n=\frac{1}{2} \operatorname{dim}_{\mathbb{F}_{p}} V$;

(2) $V=\bigcup_{i} V_{i}$ и $V_{i} \cap V_{j}=0$ для $i \neq j$;

(3) $\left\langle V_{i} \mid V_{j}\right\rangle=0$. 
Условия (1) и (2) означают, что подпространства $V_{i}$ образуют расслоение $\Sigma_{\mathrm{B}} V$. Добавляя условие (3), мы получаем симплектическое расслоение $\Sigma$.

Обратно, по указанной схеме каждому симплектическому расслоению в $V$ соответствует Ј-разложение.

В статье [4] были построены так называемые стандартнье J-разложения, для

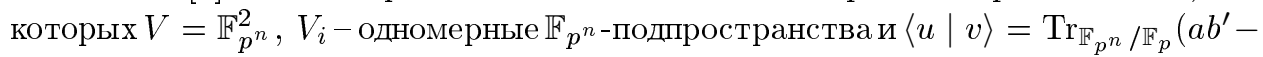
$\left.a^{\prime} b\right)$ для $u=(a ; b), v=\left(a^{\prime} ; b^{\prime}\right)$, где $\operatorname{Tr}_{\mathbb{F}_{p^{n}} / \mathbb{F}_{p}}$ обозначает функцию следа расширения поля $\mathbb{F}_{p^{n}}$ над $\mathbb{F}_{p}$.

Отметим, что термин "Ј-разложение" и приведенная выше конструкция впервые появились в статье [11]. Там же впервые были построены Ј-разложения, не сопряженные стандартньм. До сих пор не известно примеров WP-разложений, не сопряженных Ј-разложениям.

Понятие симплектического расслоения было впервые введено Кантором [12], [13]. Автор пришел к нему независимо, рассматривая WP-разложения. Кантор [12]-[14] построил множество примеров симплектических расслоений и сделал их предметом всестороннего изучения.

\section{§3. Автоморфизмы WP-разложений}

В этом параграфе мы докажем ряд технических лемм, которые затем будут использоваться при рассмотрении аналога теоремы Вагнера.

Пусть семейство подалгебр $\mathscr{D}=\left\{\mathbf{B}_{i}, i=1, \ldots, n+1\right\}$ образует WP-разложение алгебры $\mathrm{M}_{n}(\mathbb{C})=\mathrm{M}_{n}$. Положим

$$
\begin{gathered}
G=\text { Aut } \mathscr{D} ; \\
N=\left\{\sigma \in G \mid \mathbf{B}_{i}^{\sigma}=\mathbf{B}_{i}, i=1, \ldots, n+1\right\} ; \\
N_{i}=\left\{\sigma \in N \mid x^{\sigma}=x \text { для всех } x \in \mathbf{B}_{i}\right\} ; \\
T=N_{1} \cup \cdots \cup N_{n+1} .
\end{gathered}
$$

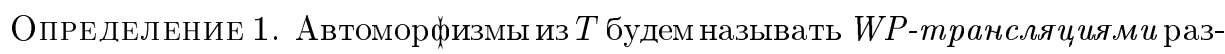
ложения $\mathscr{D}$.

Если $H \subseteq$ Aut $\mathrm{M}_{n}, X \subseteq \mathrm{M}_{n}$, то

$$
\begin{gathered}
\mathrm{St}_{H}(X)=\left\{\sigma \in H \mid x^{\sigma}=x \text { для всех } x \in X\right\}, \\
X^{H}=\left\{x^{\sigma} \mid x \in X, \sigma \in H\right\} .
\end{gathered}
$$

Как обычно, $\operatorname{tr}$ обозначает след матрицы или линейного оператора; $\operatorname{Tr}=n \cdot \operatorname{tr}-$ след матрицы в регулярном представлении; $E_{n}=E$ - единичная матрица; 1 также будет обозначать единичный элемент групшы; $\operatorname{Pr}\left(\mathbf{B}_{i}\right)$ - множество примитивных идемпотентов подалгебры $\mathbf{B}_{i}$;

$$
\operatorname{Pr}(\mathscr{D})=\operatorname{Pr}\left(\mathbf{B}_{1}\right) \cup \cdots \cup \operatorname{Pr}\left(\mathbf{B}_{n+1}\right) .
$$

Обозначим через $\chi, b, s$ характеры действий группы $G$ соответственно на $\mathrm{M}_{n}$, $\mathscr{D}, \operatorname{Pr}(\mathscr{D}) ; 1_{G}-$ единичный характер группы $G$.

$\operatorname{Conj}(X)$ обозначает сопряжение матрицей $X$. Положим

$$
G\left(J\left(p^{n}\right)\right)=\left\{\operatorname{Conj}\left(J_{u}\right), u \in \mathbb{F}_{p}^{2 n}\right\} .
$$


Лемма 1. Группа $G$ конечна.

ДокАЗАТЕльство. Множество примитивных идемпотентов коммутативной полупростой алгебры линейно порождает ее. Следовательно, идемпотенты из $\operatorname{Pr}(\mathscr{D})$ линейно порождают алгебру $\mathrm{M}_{n}$. Отсюда вытекает, что действие групшы $G$ на $\operatorname{Pr}(\mathscr{D})$ точное и, значит, $G$ конечна. Лемма доказана.

ЛЕмма 2. Справедливо соотношение $\chi=s-b+1_{G}$.

ДокАЗАТЕЛЬСтво. Пусть $b(\sigma)=b, s(\sigma)=s$. Пусть $\sigma$ нормализует подалгебры $\mathbf{B}_{i_{1}}, \ldots, \mathbf{B}_{i_{b}}$. В силу условия ортогональности имеем разложение в прямую сумму

$$
\mathrm{M}_{n}=\langle E\rangle \oplus \mathbf{B}_{1}^{\circ} \oplus \cdots \oplus \mathbf{B}_{n+1}^{\circ}
$$

где $\mathbf{B}_{i}^{\circ}=\left\{X \in \mathbf{B}_{i} \mid \operatorname{tr} X=0\right\}$. Положим

$$
V=\mathbf{B}_{i_{1}}+\cdots+\mathbf{B}_{i_{b}}, \quad I=\left\{i_{1}, \ldots, i_{b}\right\}
$$

Тогда

$$
\mathrm{M}_{n}=V \oplus W, \quad \text { где } W=\bigoplus_{j \notin I} \mathbf{B}_{j}^{\circ} .
$$

Так как $\sigma$ переставляет подпространства $\mathbf{B}_{j}^{\circ}, j \notin I$, то $\left.\operatorname{tr} \sigma\right|_{W}=0$. Далее

$$
V=\langle E\rangle \oplus \mathbf{B}_{i_{1}}^{\circ} \oplus \cdots \oplus \mathbf{B}_{i_{b}}^{\circ}
$$

Поэтому

$$
\chi(\sigma)=\operatorname{tr} \sigma=\left.\operatorname{tr} \sigma\right|_{V}=\left.\sum_{i \in I} \operatorname{tr} \sigma\right|_{\mathbf{B}_{i}}-\left.(b-1) \operatorname{tr} \sigma\right|_{\langle E\rangle} .
$$

Остается заметить, что $\left.\sum_{i \in I} \operatorname{tr} \sigma\right|_{\mathbf{B}_{i}}=s$ и $\left.\operatorname{tr} \sigma\right|_{\langle E\rangle}=1$. Лемма доказана.

В следуюшей лемме приводятся очень полезные конструкции базисов алгебры матриц $\mathrm{M}_{n}$, использу ющие примитивные идемпотенты подалгебр WP-разложения.

Лемма 3. Пусть $\mathbf{B}, \mathbf{B}^{\prime} \in \mathscr{D}, \quad \mathbf{B} \neq \mathbf{B}^{\prime} ; \quad \operatorname{Pr}(\mathbf{B})=\left\{e_{1}, \ldots, e_{n}\right\}, \quad \operatorname{Pr}\left(\mathbf{B}^{\prime}\right)=$ $\left\{f_{1}, \ldots, f_{n}\right\}$. Тогда

(1) $\operatorname{Tr} e_{i} f_{j}=1$ для всех $i, j$;

(2) матрищъ $\operatorname{Pr}(\mathbf{B}) \cdot \operatorname{Pr}\left(\mathbf{B}^{\prime}\right)=\left\{e_{i} f_{j} \mid i, j=1, \ldots, n\right\}$ образуют базис в $\mathrm{M}_{n}$;

(3) матрицъ $\operatorname{Pr}(\mathbf{B}) \cdot f \cdot \operatorname{Pr}(\mathbf{B})=\left\{e_{i} f e_{j} \mid i, j=1, \ldots, n\right\}$ образуют базис в $\mathrm{M}_{n}$ для любого $f \in \operatorname{Pr}\left(\mathbf{B}^{\prime}\right)$.

ДокАЗАТЕЛЬСтво. (1) В силу условия ортогональности имеем

$$
\operatorname{Tr}\left(e_{i}-e_{j}\right)\left(f_{k}-f_{l}\right)=0
$$

при $i \neq j, k \neq l$. Значит, $\operatorname{Tr}\left(e_{i}-e_{j}\right) f_{k}=\operatorname{Tr}\left(e_{i}-e_{j}\right) f_{l}$. Отсюда и из равенств $f_{1}+\cdots+f_{n}=E, \operatorname{Tr}\left(e_{i}-e_{j}\right)=0$ получаем

$$
\operatorname{Tr}\left(e_{i}-e_{j}\right) f_{k}=0
$$


для всех $k$. Значит, $\operatorname{Tr} e_{i} f_{k}=\operatorname{Tr} e_{j} f_{k}$. Теперь, используя равенства $e_{1}+\cdots+$ $e_{n}=E, \operatorname{Tr} f_{k}=n$, получаем требуемое.

(2) Пусть

$$
\sum_{i, j} \alpha_{i j} e_{i} f_{j}=0
$$

для некоторых коэффициентов $\alpha_{i j}$. Домножим это равенство справа на $e_{k}$, слева на $f_{l}$ и рассмотрим значение функции следа от обеих частей полученного равенства:

$$
0=\operatorname{Tr}\left(e_{k}\left(\sum_{i, j} \alpha_{i j} e_{i} f_{j}\right) f_{l}\right)=\operatorname{Tr} \alpha_{k l} e_{k} f_{l}=\alpha_{k l} .
$$

Отсюда следует, что $\left\{e_{i} f_{j}\right\}$ - линейно независимая система из $n^{2}$ элементов. Значит, $\left\{e_{i} f_{j}\right\}$ - базис в $\mathrm{M}_{n}$.

(3) Заметим, что для любых примитивных идемпотентов $a$ и $b$ алгебры матриц $\mathrm{M}_{n}$ справедливо равенство

$$
\operatorname{dim}_{\mathbb{C}} a \mathrm{M}_{n} b=1
$$

Отсюда вытекает, в частности, что $\forall x \in \mathrm{M}_{n} \exists \alpha \in \mathbb{C}: a x a=\alpha a$. Отметим, что $\alpha$ может быть найдено из условия $\operatorname{Tr} a x a=\operatorname{Tr} \alpha a$, если $\operatorname{Tr} a \neq 0$.

Используя это замечание, п. (1) и равенство $f^{2}=f$ для любого $f \in \operatorname{Pr}\left(\mathbf{B}^{\prime}\right)$, получаем

$$
1=\operatorname{Tr} e_{i} f=\operatorname{Tr} e_{i} f^{2}=\operatorname{Tr} f e_{i} f \quad \Rightarrow \quad f e_{i} f=\frac{1}{n} f
$$

для любого $e_{i} \in \operatorname{Pr}(\mathbf{B})$. Отсюда следует

$$
\operatorname{Tr} e_{i} f e_{j} f e_{i}=\frac{1}{n} \operatorname{Tr} e_{i} f e_{i}=\frac{1}{n} \operatorname{Tr} e_{i}^{2} f=\frac{1}{n} \operatorname{Tr} e_{i} f=\frac{1}{n} .
$$

Из последнего равенства вытекает $e_{i} f e_{j} \neq 0$ и, значит,

$$
\forall x \in \mathrm{M}_{n} \quad \exists \alpha_{i j} \in \mathbb{C}: e_{i} x e_{j}=\alpha_{i j} e_{i} f e_{j}
$$

для любых $e_{i}, e_{j} \in \operatorname{Pr}(\mathbf{B}), f \in \operatorname{Pr}\left(\mathbf{B}^{\prime}\right)$. Отсюда следует, что для любого $x \in \mathrm{M}_{n}$ мы имеем представление

$$
x=\left(e_{1}+\cdots+e_{n}\right) \cdot x \cdot\left(e_{1}+\cdots+e_{n}\right)=\sum_{i, j} e_{i} x e_{j}=\sum_{i, j} \alpha_{i j} e_{i} f e_{j} .
$$

Это доказывает, что матрицы $\left\{e_{i} f e_{j} \mid i, j=1, \ldots, n+1\right\}$ образуют базис в $\mathrm{M}_{n}$. Лемма доказана.

Лемма 4. Пусть $\sigma \in N \backslash T$. Тогда $\sigma$ оставляет неподвижным в каждой подалгебре из $\mathscr{D}$ ровно по одному примитивному идемпотенту. 
ДОКАЗАТЕЛЬСТво. Пусть $k_{i}$ - число неподвижных идемпотентов относительно $\sigma$ в $\operatorname{Pr}\left(\mathbf{B}_{i}\right)$. Тогда возможны два случая.

Случай 1: не более чем одно число $k_{i} \neq 0$. Тогда по лемме 2

$$
\chi(\sigma)=1+s(\sigma)-b(\sigma)=1+k_{i}-(n+1)=k_{i}-n .
$$

С другой стороны, вычисляя $\chi(\sigma)$ в базисе $\operatorname{Pr}\left(\mathbf{B}_{i}\right) \cdot \operatorname{Pr}\left(\mathbf{B}_{j}\right), i \neq j$, получаем, что

$$
\chi(\sigma)=k_{i} k_{j}=0 .
$$

Значит, $k_{i}=n$ и, следовательно, $\sigma \in N_{i} \leqslant T$. Противоречие.

Случай 2: существуют $i, j, i \neq j$, такие, что $k_{i}, k_{j} \neq 0$. Тогда для любого $s \neq i, j$ имеем

$$
k_{i} k_{s}=k_{i} k_{j}=\chi(\sigma)
$$

и, следовательно, $k_{s}=k_{j}$. Аналогично получаем $k_{s}=k_{i}$. Положим $k=k_{s}=$ $k_{i}=k_{j}$. По лемме 2

$$
k^{2}=\chi(\sigma)=1+k(n+1)-(n+1)
$$

Отсюда $k=1$ или $k=n$. Если $k=n$, то $\sigma=1$. Остается единственная возможность $k=1$. Лемма доказана.

Напомним, что группа действует на множестве полурегулярно, если каждый неединичный элемент не имеет неподвижных точек.

Лемма 5. (1) Если $\sigma$ оставляет неподвижньци подалгебру $\mathbf{B}_{i}\left(\right.$ m.е. $\left.\sigma \in N_{i}\right)$ и идемпотент $f \in \operatorname{Pr}\left(\mathbf{B}_{j}\right), i \neq j$, то $\sigma=1$;

(2) чентрализатор подалгебры $\mathbf{B}_{i}$ в группе $\mathrm{Aut}_{n} \mathrm{M}_{n}$ абелев, и, в частности, подгруппа $N_{i}$ абелева;

(3) $N_{i} \cap N_{j}=1=\left[N_{i}, N_{j}\right]$ npu $i \neq j$;

(4) $T$ - нормальная подгруппа в $G$;

(5) если при $i \neq j\left|N_{i}\right|>1,\left|N_{j}\right|>1$, то $N_{i}$ и $N_{j}$ - әлементарные абелевы p-группы;

(6) $N_{i}$ действует полурегулярно на $\operatorname{Pr}\left(\mathbf{B}_{j}\right)$ для любого $j \neq i$;

(7) $\left|N_{i}\right| \leqslant n$ для всех $i \quad u|T| \leqslant n^{2}$.

ДоказАТЕЛЬСтво. (1) В силу леммы $3 \operatorname{Pr}\left(\mathbf{B}_{i}\right) \cdot f \cdot \operatorname{Pr}\left(\mathbf{B}_{i}\right)$ - базис в $\mathrm{M}_{n}$. Так как по условию $\sigma$ оставляет неподвижньми элементы этого базиса, то $\sigma=1$.

(2) В базисе $\operatorname{Pr}\left(\mathbf{B}_{i}\right) \cdot f \cdot \operatorname{Pr}\left(\mathbf{B}_{i}\right)$ централизатор подалгебры $\mathbf{B}_{i}$ представляется диагональными матрицами, поэтому он абелев.

(3) В силу п. (1) $N_{i} \cap N_{j}=1$. Пусть теперь $\sigma \in N_{i}, \tau \in N_{j}$, тогда, рассматривая действие $\sigma \tau$ и $\tau \sigma$ на базисе $\operatorname{Pr}\left(\mathbf{B}_{i}\right) \cdot \operatorname{Pr}\left(\mathbf{B}_{j}\right)=\left\{e_{k} f_{l} \mid e_{k} \in \operatorname{Pr}\left(\mathbf{B}_{i}\right), f_{l} \in \operatorname{Pr}\left(\mathbf{B}_{j}\right)\right\}$, получаем

$$
\left(e_{k} f_{l}\right)^{\sigma \tau}=e_{k}^{\tau} f_{l}^{\sigma}=\left(e_{k} f_{l}\right)^{\tau \sigma}
$$

Значит, $\left[N_{i}, N_{j}\right]=1$.

(4) Очевидно, что $N$ - нормальная подгруппа в $G$. Так как сопряженные в $G$ автоморфизмы имеют одинаковое количество неподвижных примитивных идемпотентов в $\operatorname{Pr}(\mathscr{D})$ (и одинаковое значение характера $\chi$ ), то по лемме 4 множество $T=N_{1} \cup \cdots \cup N_{n+1}$ нормально в $G$. Покажем, что $T$ - подгруппа. Пусть $\sigma \in N_{i}$, 
$\tau \in N_{j}$. Тогда автоморфизм $\sigma \tau$ не имеет неподвижных точек как в $\operatorname{Pr}\left(\mathbf{B}_{i}\right)$, так и в $\operatorname{Pr}\left(\mathbf{B}_{j}\right)$. Поэтому в силу леммы $4 \sigma \tau \notin N \backslash T$, т.е. $\sigma \tau \in T$.

(5) Пусть $\sigma \in N_{i}, \tau \in N_{j}$, ord $\sigma=n_{1}>1$, ord $\tau=n_{2}>1$. Тогда $\sigma \tau$ действует без неподвижных точек на $\operatorname{Pr}\left(\mathbf{B}_{i}\right)$ и на $\operatorname{Pr}\left(\mathbf{B}_{j}\right)$. Значит, в силу леммы $4 \sigma \tau \in N_{s}$ для некоторого $s \neq i, j$. В силу п. (3) $\sigma \tau=\tau \sigma$, поэтому

$$
(\sigma \tau)^{n_{1}}=\tau^{n_{1}}=1
$$

где последнее равенство вытекает из того, что $\tau^{n_{1}}$ действует тождественно на $\operatorname{Pr}\left(\mathbf{B}_{s}\right)$ и на $\operatorname{Pr}\left(\mathbf{B}_{j}\right)$. Аналогично получаем

$$
(\sigma \tau)^{n_{2}}=\sigma^{n_{2}}=1
$$

Далее выводим, что

$$
\tau^{n_{1}}=1, \quad \text { ord } \tau=n_{2} \quad \Rightarrow \quad n_{2} \text { делит } n_{1}
$$

и, аналогично,

$$
\sigma^{n_{2}}=1, \quad \text { ord } \sigma=n_{1} \Rightarrow n_{2} \text { делит } n_{1} .
$$

Таким образом, $n_{1}$ и $n_{2}$ делят друг друга, т.е. $n_{1}=n_{2}$. Отсюда следует, что порядки всех неединичных элементов в $N_{i}$ и $N_{j}$ равны. Так как в каждой конечной группе есть элемент простого порядка, то $N_{i}$ и $N_{j}$ - элементарные абелевы (в силу п. (2)) $p$-группы для некоторого простого $p$.

Пункт (6) следует из п. (1).

(7) В силу п. (6) $\left|N_{i}\right| \leqslant n$. Поэтому

$$
|T|=\left|N_{1} \cup \cdots \cup N_{n+1}\right| \leqslant(n-1)(n+1)+1=n^{2} .
$$

Лемма доказана.

ОПРЕДЕЛЕНИЕ 2 . Группу $T$ будем назьвать группой $W P$-трансляций разложения $\mathscr{D}$.

Лемма 6. Пусть для некоторой подалгебры $\mathbf{B}_{i} \in \mathscr{D}$ множество ее примитивных идемпотентов $\operatorname{Pr}\left(\mathbf{B}_{i}\right)$ лежит в $G$-орбите. Тогда если $|N|>1$, то $|T|>1$.

ДокаЗАТЕЛЬСтво. Пусть $T=1$, тогда $N_{i}=1$ и $N$ действует на $\operatorname{Pr}\left(\mathbf{B}_{i}\right)$ точно с характером $\theta$ степени $n=\left|\operatorname{Pr}\left(\mathbf{B}_{i}\right)\right|$. В силу леммы $4 \theta(x)=1$ для всех $x \in N \backslash\{1\}$. Отсюда следует, что характер $\theta$ можно представить в виде

$$
\theta=1_{N}+a \rho_{N},
$$

где $\rho_{N}$ - регулярный характер группы $N$ и коэффициент $a=(n-1) /|N|$ находится из рассмотрения степеней характеров. Так как $\theta-1_{N}$ является обобщенным характером группы $N$, то $a$ должно быть целым, ибо $\rho_{N}$ содержит единичный характер $1_{N}$ с кратностью 1 . Поэтому $|N|$ делит $n-1$.

С другой стороны, так как подгруппа $N$ нормальна в $G$, то $N$ действует полутранзитивно на $\operatorname{Pr}\left(\mathbf{B}_{i}\right)$, т.е. все $N$-орбиты имеют одинаковую длину. Значит, $|N|$ делит $n$. Отсюда $|N|=1$. Противоречие доказывает лемму.

Следующая лемма близка по духу к теореме 4.26 из работы [2] и следствию 1 из нее, принадлежашему Глисону, см. также [15; теорема 20.9.6] и [16]. 
Лемма 7. Пусть $\left|N_{i}\right|=k>1$ для всех $i$. Тогда $\left|N_{i}\right|=n$ для любого $i$.

ДоказАтельство. Пусть $e \in \operatorname{Pr}\left(\mathbf{B}_{i}\right)$. В силу леммы 5, п. (1) $\operatorname{St}_{T}(e)=N_{i}$. Кроме того,

$$
|T|=\left|\operatorname{St}_{T}(e)\right| \cdot\left|e^{T}\right| \text {. }
$$

Отсюда $\left|e^{T}\right|=\left|f^{T}\right|=s$ для любого $f \in \operatorname{Pr}\left(\mathbf{B}_{i}\right)$. Значит, длина любой $T$-орбиты $s$ делит $n$ и, следовательно, $|T|=k s$ делит $k n$.

С другой стороны, по условию

$$
|T|=1+(n+1)(k-1)=k n+k-n
$$

Отсюда $k n+k-n$ делит $k n$. Следовательно, $k-n=0$ или $n-k \geqslant k n / 2$. Но $k>1$, значит, $k-n=0$. Лемма доказана.

Лемма 8. Пусть $|T|=n^{2}$, тогда неподвижными относительно $T$ будут только скалярные матрицьь.

ДокаЗАТЕЛЬСтво. Пусть $\operatorname{Pr} \mathbf{B}_{i}=\left\{e_{i 1}, \ldots, e_{i n}\right\}, i=1, \ldots, n+1$. Тогда каждая матрица $X$ однозначно записывается в виде

$$
X=\sum_{i, j} \alpha_{i j} e_{i j}, \quad \text { где } \alpha_{1 i}+\cdots+\alpha_{n i}=\mathrm{const}
$$

для всех $i=1, \ldots, n+1$. Так как $T$ транзитивна на каждом множестве $\operatorname{Pr}\left(\mathbf{B}_{i}\right)$, то неподвижность $X$ относительно $T$ влечет равенство коэффициентов $\alpha_{i j}=\alpha_{k l}$ для любых $i, j, k, l$. Это означает, что $X=\lambda E$. Лемма доказана.

ЛЕмма 9. Пусть $\mathscr{D}$ - J-разложсение алгебрь $\mathrm{M}_{p^{n}}$, moгда групnа Aut $\mathscr{D}$ coдержит нормальную әлементарную абелеву р-подгруппу

$$
G\left(J\left(p^{n}\right)\right)=\left\{\operatorname{Conj}\left(J_{u}\right) \mid u \in \mathbb{F}_{p}^{2 n}\right\}
$$

порядка $p^{2 n}=\operatorname{dim} \mathrm{M}_{p^{n}}$. При этом $G\left(J\left(p^{n}\right)\right)$ действует тожсдественно на $\mathscr{D}$ (т.е. переводит в себя каждую подалгебру из $\mathscr{D})$.

ДокАЗАТЕльство. Так как $J_{u} J_{v} J_{u}^{-1}=\varepsilon^{\langle u \mid v\rangle} J_{v}$, то сопряжения матрицами $J_{u}$ являются автоморфизмами $J$-разложения $\mathscr{D}$, при этом они оставляют инвариантными каждую подалгебру из $\mathscr{D}$, т.е. группа $G\left(J\left(p^{n}\right)\right)$ действует тривиально на множестве подалгебр $\mathscr{D}$. Значит, $G\left(J\left(p^{n}\right)\right)$ нормальна в Aut $\mathscr{D}$. Лемма доказана.

ЗАмЕчАниЕ. Позже (см. следствие 1$)$ мы докажем, что $G(J(n))$ является группой WP-трансляций любого J-разложения алгебры $\mathrm{M}_{n}$.

Лемма 10. Пусть группа WP-трансляиий $T=N_{1} \cup \cdots \cup N_{p^{n}+1}$ WP-разложения $\mathscr{D}=\left\{B_{i}\right\}$ алгебры матрии $\mathrm{M}_{p^{n}}$ совпадает с группой $G\left(J\left(p^{n}\right)\right)$. Тогда D является J-разложением.

ДоказАтельство. Пусть $N_{i}=\left\{\operatorname{Conj}\left(J_{u}\right) \mid u \in V_{i}\right\}$. Так как подалгебра $\mathbf{B}_{i}$ совпадает со своим централизатором в алгебре $\mathrm{M}_{n}$, то $N_{i} \subset \mathbf{B}_{i}$. Следовательно, $\mathbf{B}_{i}=\left\langle J_{u} \mid u \in V_{i}\right\rangle_{\mathbb{C}}$ и $\mathscr{D}$ является Ј-разложением. Лемма доказана. 
Лемма 11. Пусть $|T|=n^{2}$, тогда $n=p^{m}$, где $p$ простое, и $T$ - әлементарная абелева р-группа, сопряженная в $\mathrm{Aut}_{p^{m}}$ с группой $G\left(J\left(p^{m}\right)\right)$. При әтом WP-разложение $\mathscr{D}$ сопряжено Ј-разложению.

ДокаЗАТельство. Так как $N_{i}$ в силу леммы 5 , п. (1) действует на примитивных идемпотентах подалгебры $\mathbf{B}_{j}$ полурегулярно при $i \neq j$, то $\left|N_{i}\right| \leqslant n$. Поэтому в $T$ сушествуют две неединичные подгрупшы $N_{i}$ и $N_{j}$. В силу леммы 5, п. (5) любая подгруппа $N_{s}$ является элементарной абелевой $p$-группой для некоторого простого $p$. Следовательно, группа $T=N_{1} \cup \cdots \cup N_{n+1}$ - элементарная абелева $p$-группа и $n=p^{m}$.

Как установлено в [17], конечные неприводимые абелевы подгруппы в Aut $\mathrm{M}_{n}$ сопряжены в Aut $\mathrm{M}_{n}$ тогда и только тогда, когда они изоморфны. Значит, $T$ сопряжена в Aut $\mathrm{M}_{p^{m}}$ с группой $G\left(J\left(p^{m}\right)\right)$. Следовательно, $\mathscr{D}$ сопряжено с WP-разложением $\mathscr{D}^{\prime}$, группа автоморфизмов которого содержит $G\left(J\left(p^{m}\right)\right)$. В силу леммы 10 $\mathscr{D}^{\prime}$ является Ј-разложением. Лемма доказана.

\section{§4. Аналог теоремы Вагнера}

Каждое расслоение $\Sigma=\left\{V_{i}\right\}$ (см. $\left.\S 2\right) \mathbb{F}_{q}$-пространства $V$ определяет аффинную плоскость $\mathscr{A}(\Sigma)$ специального вида - так называемую плоскость трансляций, точками которой являются векторы пространства $V$, а прямыми - смежные классы подпространств $V_{i}$. При этом выполняются следующие аксиомы, определяющие афффинную плоскость:

(1) каждая прямая содержит по крайней мере две точки;

(2) через любые две точки проходит единственная прямая;

(3) для любой прямой $L$ и точки $v \notin L$ существует единственная прямая, содержашая $v$ и не пересекаюшая $L$;

(4) сушествуют три неколлинеарные точки.

Для любого вектора $u \in V$ отображение $v \mapsto v+u$ является коллинеациeй (т.е. сохраняет отношение инцидентности точка-прямая) плоскости $\mathscr{A}(\Sigma)$, при этом каждый класс параллельных прямых переходит в себя. Коллинеация $\alpha$ аффинной плоскости $\mathscr{A}$ называется трансляцией, если $\alpha$ действует тривиально на множестве классов параллельных прямых и действует "без неподвижных точек" на множестве точек плоскости $\mathscr{A}$. Хорошо известно (см. [2]), что трансляции могут быть охарактеризованы следуюшим условием:

$(\star)$ если коллинеация $\alpha$ действует тривиально на множестве классов параллельных прямых и для каждого класса параллельности $\alpha$ действует на множестве его прямых либо "без неподвижных точек", либо тождественно, то $\alpha$ является трансляцией.

Множество всех трансляций образует группу $T$, которая действует полурегулярно на множестве точек и которая нормальна в полной группе коллинеаций плос-

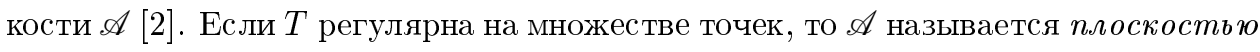
трансляций, а $T$ при этом называется группой трансляций плоскости $\mathscr{A}$. Этому условию удовлетворяет плоскость $\mathscr{A}(\Sigma)$.

Каждая прямая аффинной плоскости $\mathscr{A}$ содержит одинаковое число точек, которое называется порядком плоскости $\mathscr{A}$. Плоскость порядка $n$ содержит $n^{2}$ точек. 
Следовательно, если $\mathscr{A}$ - плоскость трансляпий порядка $n$, то ее группа трансляций $T$ содержит $n^{2}$ элементов.

Нам понадобится следуюшая хорошо известная (см. [2]) характеризация плоскостей трансляций:

$(\star \star)$ аффинная плоскость $\mathscr{A}$ является плоскостью трансляций, если ее группа трансляций действует транзитивно на множестве прямых каждого класса параллельности.

Плоскости трансляций представляют для нас особенный интерес. Знаменитая теорема Вагнера утверждает, что конечная аффинная плоскость, обладающая транзитивной на прямых группой коллинеаций, является плоскостью трансляций [1].

Напомним соответствие между аффинными плоскостями порядка $n$ и однородньгми ОР типа $n \mathrm{M}_{1}$ алгебры $n^{2} \mathrm{M}_{1}$ : идемпотенты $\operatorname{Pr}\left(n^{2} \mathrm{M}_{1}\right)$ рассматриваются как точки, а идемпотенты $\operatorname{Pr}(\mathscr{D})$ - как прямые, где $\mathscr{D}-\mathrm{OP}$ типа $n \mathrm{M}_{1}$. В этой ситуации подалгебрам из $\mathscr{D}$ соответствуют классы параллельности.

Теперь мы можем переформулировать теорему Вагнера в терминах теории ОР:

TеОРема ВаГнеРА. Пусть $\mathscr{D}$ - однородное OP muna $n \mathrm{M}_{1}$ алzебры $n^{2} \mathrm{M}_{1}$. Eсли Aut $\mathscr{D}$ действует транзитивно на $\operatorname{Pr}(\mathscr{D})$, то Aut $\mathscr{D}$ содержит нормальную әлементарную абелеву подгруппу, регулярную на $\operatorname{Pr}\left(n^{2} \mathrm{M}_{1}\right)$ и тождественную на $\mathscr{D}$ (другими словами, $\mathscr{D}$ соответствует плоскости трансляций).

Вернемся к WP-разложениям и обсудим более подробно их связь с аффинными плоскостями. Следуя указанному выше соответствию между аффинными плоскостями порядка $n$ и однородными $\mathrm{OP}$ типа $n \mathrm{M}_{1}$ алгебры $n^{2} \mathrm{M}_{1}$, мы видим, что прямым на плоскости соответствуют примитивные идемпотенты подалгебр ортогонального разложения, причем классы параллельности отвечают самим подалгебрам.

Следуюшая лемма ввиду характеризации ( $\star$ оправдывает термин "WP-трансляпия".

Лемма 12. Пусть автоморфизм а является WP-трансляцией WP-разложения Д. Тогда

(1) $\alpha$ действует тождественно на $\mathscr{D}$;

(2) для любой подалгебрь $\mathbf{B} \in \mathscr{D}$ $\alpha$ действует на $\operatorname{Pr}(\mathbf{B})$ либо тождественно, либо "без неподвижных точек".

ДокАЗАТЕЛЬСтво. Пункт (1) вытекает из определений.

(2) Пусть $\alpha \in N_{i}$, т.е. $\alpha$ действует на $\operatorname{Pr}\left(\mathbf{B}_{i}\right)$ тождественно. Тогда в силу леммы 5 , п. (1) на множестве примитивных идемпотентов любой другой подалгебры из $\mathscr{D} \alpha$ действует “без неподвижных точек”. Лемма доказана.

Лемма 13. Пусть автоморфизм $\alpha \in$ Aut $\mathscr{D}$ действует тривиально на $\mathscr{D}$. Тогда а является WP-транслячией тогда и только тогда, когда $\alpha$ есть сопряжсние матричей с нулевым следом.

ДокАЗАТЕльство. Будем использовать обозначения $\S 3$. В силу леммы 2 имеем соотношение для характеров группы $G=$ Aut $\mathscr{D}$ :

$$
\chi=s-b+1_{G} .
$$


Пусть $\alpha$ является сопряжением матрицей $X$. В силу конечности группы $G$ (по лемме 1) автоморфизм $\alpha$ имеет конечный порядок. Поэтому мы можем предполагать, что матрица $X$ также имеет конечный порядок. В этом случае $\operatorname{tr} X^{-1}=\overline{\operatorname{tr} X}$.

$\Leftarrow$. Пусть $\operatorname{tr} X=0$. Тогда

$$
\chi(\alpha)=\operatorname{tr} X \cdot \operatorname{tr} X^{-1}=0 .
$$

Так как $\alpha$ действует тождественно на $\mathscr{D}$, то $b(\alpha)=n+1$. Следовательно, $s(\alpha)=n$. Это означает, что $\alpha$ имеет $n$ неподвижных примитивных идемпотентов в $\operatorname{Pr}(\mathscr{D})$. Если любые два из этих идемпотентов принадлежат различным подалгебрам $\mathbf{B}_{i}, \mathbf{B}_{j} \in \mathscr{D}$, то, рассматривая базис $\operatorname{Pr}\left(\mathbf{B}_{i}\right) \cdot \operatorname{Pr}\left(\mathbf{B}_{j}\right)$ в $\mathrm{M}_{n}$, мы получаем $\chi(\alpha) \neq 0$. Это противоречит формуле (1). Значит, все неподвижные идемпотенты автоморфизма $\alpha$ лежат в одной подалгебре и, следовательно, $\alpha$ является WP-трансляцией.

$\Rightarrow$. Пусть $\alpha$ является WP-трансляцией. Рассматривая действие $\alpha$ на базисе $\operatorname{Pr}\left(\mathbf{B}_{i}\right) \cdot \operatorname{Pr}\left(\mathbf{B}_{j}\right)$ для любых двух различных подалгебр, мы получаем $\chi(\alpha)=0$. Теперь из формулы (1) следует

$$
\operatorname{tr} X \cdot \operatorname{tr} X^{-1}=\operatorname{tr} X \cdot \overline{\operatorname{tr} X}=|\operatorname{tr} X|^{2}=0 .
$$

Значит, $\operatorname{tr} X=0$. Лемма доказана.

СлеДСтвИЕ 1. (1) Сопряжсение матрицей $J_{u}$ является WP-трансляцией любого Ј-разложения.

(2) Группа $G(J(n))$ является группой WP-трансляций любого J-разложения.

ДокаЗАТЕЛЬСтво. (1) Так как $\operatorname{tr} J_{u}=0$, если $u \neq 0$, то с учетом леммы 9 из леммы 13 следует, что $\operatorname{Conj}\left(J_{u}\right)$ является WP-трансляцией.

(2) Пусть $T$ - группа WP-трансляций J-разложения $\mathscr{D}$ алгебры $\mathrm{M}_{n}$. B силу леммы 5, п. (7) $|T| \leqslant n^{2}$. Так как $|G(J(n))|=n^{2}$, то в силу п. (1) $G(J(n))=T$. Следствие доказано.

Осталось определить аналог плоскостей трансляций. Так как для WP-разложений нет подходящего аналога множества точек аффинной плоскости, то мы будем опираться на характеризацию $(\star \star)$ аффинных плоскостей.

ЛЕмма 14. Пусть $T$ - группа WP-трансляций WP-разложения $\mathscr{D}=\left\{\mathbf{B}_{i}\right\}$ алгебры матрии $\mathrm{M}_{n}$. Тогда следующие условия эквивалентны:

(1) для каждой подалгебры $\mathbf{B}_{i} T$ действует транзитивно на множсестве ее примитивных идемпотентов $\operatorname{Pr}\left(\mathbf{B}_{i}\right)$;

(2) Т является әлементарной абелевой р-группой порядка $n^{2}$;

(3) $T$ сопряжсено $G(J(n))$;

(4) D сопряжсено J-разложению.

ДокАЗАТЕЛЬСтво. (1) $\Rightarrow(2)$. Пусть

$$
\left|N_{i}\right|=k=\max \left\{\left|N_{j}\right|, j=1, \ldots, n+1\right\} .
$$

Тогда

$$
|T|=\left|N_{1} \cup \cdots \cup N_{n+1}\right| \leqslant(k-1)(n+1)+1 .
$$


Подгруппа $N_{i}$ является ядром действия группы $T$ на множествепримитивных идемпотентов $\operatorname{Pr}\left(\mathbf{B}_{i}\right)$. Так как $T$ действует на $\operatorname{Pr}\left(\mathbf{B}_{i}\right)$ транзитивно, то

$$
\left|T: N_{i}\right| \geqslant n
$$

Таким образом, мы имеем неравенство

$$
n \leqslant|T|: k \leqslant \frac{(k-1)(n+1)+1}{k} .
$$

Отсюда получаем $k \geqslant n$ и, значит, $k=n$. Следовательно,

$$
|T| \geqslant k n \geqslant n^{2} .
$$

В силу леммы 5 , п. (7) $|T| \leqslant n^{2}$. Значит, $|T|=n^{2}$. В силу леммы $11 n=p^{m}$, где п простое.

Импликация $(2) \Rightarrow(3)$ вытекает из леммы 11.

Импликация $(3) \Rightarrow(4)$ вытекает из леммы 10.

$(4) \Rightarrow(1)$. В силу следствия 1, п. (2) группа $G(J(n))$ является группой трансляций Ј-разложения $\mathscr{D}$. Имеем, что $\left|N_{i}\right|=n$ и в силу леммы 5 , п. (6) $N_{i}$ действует полурегулярно на множестве $\operatorname{Pr}\left(\mathbf{B}_{j}\right)$ при $j \neq i$. Значит, $N_{i}$ действует на $\operatorname{Pr}\left(\mathbf{B}_{j}\right)$ регулярно для любых $i, j, i \neq j$. Следовательно, $T$ действует на каждом множестве $\operatorname{Pr}\left(\mathbf{B}_{i}\right)$ транзитивно. Лемма доказана.

Таким образом, группа $G(J(n))$ является естественньм аналогом группы трансляций. Следовательно, плоскостям трансляций отвечают Ј-разложения. Суммируем полученные выводы в таблице:

\begin{tabular}{|l|l|}
\hline афффинная плоскость $\mathscr{A}$ порядка $n$ & WР-разложение $\mathscr{D}$ алгебры $\mathrm{M}_{n}$ \\
\hline количество точек в $\mathscr{A}$ & размерность алгебры $\mathrm{M}_{n}$ \\
\hline множество прямых в $\mathscr{A}$ & $\operatorname{Pr}(\mathscr{D})$ \\
\hline класс параллельности & $\operatorname{Pr}(\mathbf{B})$ для $\mathbf{B} \in \mathscr{D}$ \\
\hline трансляция & WP-трансляция \\
\hline плоскость трансляций & Ј-разложение \\
\hline
\end{tabular}

Установленное соответствие позволяет сформулировать аналог теоремы Вагнера для WP-разложений.

ГипотезА 1 (аналог теоремы Вагнера). Если WP-разложсение $\mathscr{D}$ алъебры $\mathrm{M}_{n}$ допускает группу автоморфизмов, транзитивную на $\operatorname{Pr}(\mathscr{D})$, то $\mathscr{D}$ сопряжено Ј-разложению и, в частности, Aut $\mathscr{D}$ содержит неприводимую нормальную әлементарную абелеву подгруппу порядка $n^{2}=p^{2 m}, \quad p$ простое, действующую тождественно на $\mathscr{D}$.

Следуюшая теорема - центральная в статье. Она является аналогом теоремы Глисона (см. следствие 1 к теореме 4.26 из [2] и теорему 20.9.6 из [15], а также [16]). 
TeOpema 1 [5]. Пусть $\mathscr{D}$ - WP-разложение и Aut $\mathscr{D}$ действует транзитивно на $\operatorname{Pr}(\mathscr{D})$. Если Aut $\mathscr{D}$ содержит неединичный автоморфизм, действующий тождественно на $\mathscr{D}$, то $\mathscr{D}$ сопряжено Ј-разложению.

ДокаЗАтельство. По условию группа $N=\left\{\sigma \in\right.$ Aut $\mathscr{D} \mid \mathbf{B}^{\sigma}=\mathbf{B}$ для всех $\sigma \in \mathscr{D}\}$ нетривиальная. Значит, в силу леммы 6 группа трансляций $T$ также нетривиальная.

Очевидно, что Aut $\mathscr{D}$ действует транзитивно на множестве подалгебр WP-разложения $\mathscr{D}$. Отсюда следует, что все подгруппы $N_{i}=\left\{\sigma \in N \mid x^{\sigma}=x\right.$ для всех $\left.x \in \mathbf{B}_{i}\right\}$ имеют одинаковый порядок $k$. Так как $|T|=\left|N_{1} \cup \cdots \cup N_{n+1}\right|>1$, то $k>1$. Из леммы 7 следует, что $k=n$ и, значит, $|T|=n^{2}$. Теперь в силу леммы 14 получаем, что $\mathscr{D}$ сопряжено Ј-разложению. Теорема доказана.

Следуюшая, вторая по важности теорема этого параграфа подтверждает гипотезу 1 для алгебр матриц $\mathrm{M}_{n}$, когда $n$ нечетно и не является квадратом.

Tеорема 2 [5]. Пусть $\mathscr{D}$ - WP-разложсение алгебры $\mathrm{M}_{n}, \quad n$ - нечетный неквадрат. Если Aut $\mathscr{D}$ действует транзитивно на $\operatorname{Pr}(\mathscr{D})$, то $\mathscr{D}$ сопряхсено Ј-разложению.

ДокАЗАТЕльСтво. Положим $G=\operatorname{Aut} \mathscr{D}$. Из транзитивности $G$ на множестве примитивных идемпотентов $\operatorname{Pr}(\mathscr{D})$ следует, что порядок $G$ делится на $n(n+1)=$ $|\operatorname{Pr}(\mathscr{D})|$. Значит, порядок групшы $G$ четен. Пусть $1 \neq \sigma \in G$ и $\sigma^{2}=1$. Если $\mathbf{B}_{i}^{\sigma}=\mathbf{B}_{j}, \quad i \neq j$, то $\sigma=\operatorname{Conj}(A)$ для некоторой инволютивной подходящей матрицы $A=\left(a_{i j}\right)$ :

$$
\left(a_{i j}\right) \cdot\left(b_{i j}\right)=n E, \quad b_{i j}=a_{j i}^{-1}, \quad A^{2}=E .
$$

Отсюда $a_{i j} \cdot a_{j i}=\frac{1}{n}$ для любых $i, j$. В частности,

$$
a_{i i}^{2}=\frac{1}{n} \quad \text { и, значит, } \quad a_{i i}= \pm \frac{1}{\sqrt{n}} .
$$

Так как след инволютивной матрицы всегда целое число и $n$ не является квадратом, то

$$
\operatorname{tr} A=\sum_{i} a_{i i}=0 .
$$

Но последнее равенство невозможно ввиду нечетности $n$. Таким образом, мы получили $\mathbf{B}^{\sigma}=\mathbf{B}$ для всех $\mathbf{B} \in \mathscr{D}$. Значит, $|N|>1$. Далее применяем теорему 1. Теорема доказана.

\section{§5. Неприводимые WP-разложения}

ОПРЕДЕЛЕниЕ 3. WP-разложение $\mathscr{D}$ называется неприводимыц, если Aut $\mathscr{D}$ действует неприводимо на линейном пространстве матриц с нулевым следом.

Пусть $\mathscr{D}=\left\{\mathbf{B}_{1}, \ldots, \mathbf{B}_{n+1}\right\}-\mathrm{WP}$-разложение алгебры матриц $\mathrm{M}_{n}, \operatorname{Pr}\left(\mathbf{B}_{i}\right)=$ $\left\{e_{i 1}, \ldots, e_{i n}\right\}, i=\overline{1, n+1}$. Положим $G=\operatorname{Aut} \mathscr{D} ; G_{i}$ - нормализатор подалгебры $\mathbf{B}_{i}$ в $G ; G_{i, j}=G_{i} \cap G_{j} ; G_{i s}-$ стабилизатор в $G$ идемпотента $e_{i s} ; G_{i s, j}=$ $G_{i s} \cap G_{j}$. Положим $\tau=\chi-1_{G}$. В силу леммы 2 имеем

$$
s=\tau+b
$$

Неприводимость WP-разложения $\mathscr{D}$ означает неприводимость характера $\tau$. 
Лемма 15. Пусть $\mathscr{D}$ - неприводимое WP-разложсение алгебры $\mathrm{M}_{n}$. Тогда для всех $s=\overline{1, n}, \quad i, j=\overline{1, n+1}, \quad i \neq j$ :

(1) $G_{i}$ действует 2-транзитивно на $\operatorname{Pr}\left(\mathbf{B}_{i}\right)$;

(2) $G_{i s, j}$ действует транзитивно на $\operatorname{Pr}\left(\mathbf{B}_{j}\right)$;

(3) $G_{i, j}$ действует транзитивно на множсестве упорядоченных пар $\operatorname{Pr}\left(\mathbf{B}_{i}\right) \times \operatorname{Pr}\left(\mathbf{B}_{j}\right)$.

ДокАЗАтЕльство. Неприводимость группы $G$ на пространстве матриц с нулевьм следом влечет транзитивность $G$ на множестве примитивных идемпотентов $\operatorname{Pr}(\mathscr{D})$. Поэтому характер подстановочного представления группы $G$ на множестве $\operatorname{Pr}(\mathscr{D})$ индуцируется единичным характером стабилизатора примитивного идемпотента: $\left(1_{G_{i s}}\right)^{G}=s$.

Так как для $n=2$ WP-разложение единственно с точностью до сопряженности [4] и для него утверждения леммы 15 легко проверяются непосредственно, то далее будем считать $n>2$.

Орбиты действия группы $G_{i s}$ на множестве подалгебр $\mathscr{D}=\left\{\mathbf{B}_{i}\right\}$ будем называть $b$-орбитами, а орбиты действия $G_{i s}$ на множестве примитивных идемпотентов $\operatorname{Pr}(\mathscr{D})$ будем называть $s$-орбитами.

(1), (2) Так как $\left(1_{G_{i s}}\right)^{G}=s$, то в силу формулы (2) и формулы взаимности Фробениуса [18]

$$
\left[s_{G_{i s}}, 1_{G_{i s}}\right]-\left[b_{G_{i s}}, 1_{G_{i s}}\right]=\left[\tau_{G_{i s}}, 1_{G_{i s}}\right]=\left[\tau,\left(1_{G_{i s}}\right)^{G}\right]=[\tau, s] .
$$

Так как $\tau(1)=n^{2}-1>n+1=b(1)$ при $n>2$, то в силу неприводимости $\tau$ имеем $[\tau, b]=0$. Значит, $[\tau, s]=[\tau, \tau]=1$. Отсюда получаем $\left[s_{G_{i s}}, 1_{G_{i s}}\right]-\left[b_{G_{i s}}, 1_{G_{i s}}\right]=1$, т.е. число $s$-орбит на 1 больше числа $b$-орбит. Ясно, что каждая $s$-орбита содержится в семействе подалгебр, лежащих в одной $b$-орбите. Так как подалгебра $\mathbf{B}_{i}$, являюшаяся $b$-орбитой, содержит по крайней мере две $s$-орбиты, одна из которых состоит из неподвижного примитивного идемпотента $e_{i s}$, то подалгебры каждой из оставшихся $b$-орбит содержат ровно по одной $s$-орбите и $\mathbf{B}_{i}$ содержит ровно две $s$-орбиты. Следовательно, $G_{i}$ действует 2-транзитивно на $\operatorname{Pr}\left(\mathbf{B}_{i}\right)$ и $G_{i s, j}$ действует транзитивно на $\operatorname{Pr}\left(\mathbf{B}_{j}\right)$ для всех $j, i \neq j$.

(3) Из п. (2) следует, что $G_{i, j}$ действует транзитивно на $\operatorname{Pr}\left(\mathbf{B}_{j}\right)$ и в силу произвольности выбора $i, j$ - на $\operatorname{Pr}\left(\mathbf{B}_{i}\right)$. Еше раз применяя п. (2), получаем транзитивность $G_{i, j}$ на множестве упорядоченных пар $\operatorname{Pr}\left(\mathbf{B}_{i}\right) \times \operatorname{Pr}\left(\mathbf{B}_{j}\right)$. Лемма доказана.

ЛЕмма 16. Пусть группа $H$ действует транзитивно на множестве $\Omega$, $|\Omega|=p^{n}$, р простое. Тогда каждая силовская р-подгруппа группь $H$ также действует на $\Omega$ транзитивно.

ДокАЗАтЕльство. Пусть $P$ - силовская $p$-подгруппа группы $H,|P|=p^{n+k}$. Пусть $H_{\alpha}, P_{\alpha}$ - стабилизаторы точки $\alpha \in \Omega$ соответственно в $H$ и в $P, \alpha^{P}-P$-орбита. Тогда в силу транзитивности $H|H|=p^{n} \cdot\left|H_{\alpha}\right|$. Следовательно, порядок силовской $p$-подгрупшы стабилизатора $H_{\alpha}$ равен $p^{k}$. Далее

$$
|P|=\left|P_{\alpha}\right| \cdot\left|\alpha^{P}\right|, \quad\left|\alpha^{P}\right| \leqslant|\Omega|=p^{n} \Rightarrow\left|P_{\alpha}\right|=|P| /\left|\alpha^{P}\right| \geqslant p^{k} .
$$

Но $P_{\alpha}$ содержится в некоторой силовской $p$-подгруппе стабилизатора $H_{\alpha}$. Значит, $\left|P_{\alpha}\right|=p^{k}$ и $\left|\alpha^{P}\right|=p^{n}$, т.е. $P$ действует на $\Omega$ транзитивно. Лемма доказана. 
Tеорема 3. Пусть $\mathscr{D}=\left\{\mathbf{B}_{1}, \ldots, \mathbf{B}_{n+1}\right\}$ - неприводимое WP-разложение алгебры $\mathrm{M}_{n}, n=p^{m}$, р простое. Тогда $\mathscr{D}$ сопряжено Ј-разложению.

ДокАЗАТЕЛЬСТво. Без ограничения общности можем считать, что силовская $p$-подгруппа $P$ группы $G_{1,2}$ имеет максимальньй порядок среди силовских $p$-подгрупп групा $G_{i, j}, i \neq j$. В силу лемм $15,16 P$ действует транзитивно на $\operatorname{Pr}\left(\mathbf{B}_{1}\right) \times$ $\operatorname{Pr}\left(\mathbf{B}_{2}\right)$.

Рассмотрим центральньй автоморфизм $z \in Z(P), z \neq 1$. Множество $I_{z}$ неподвижных относительно $z$ примитивных идемпотентов в $\operatorname{Pr}(\mathscr{D}) \quad P$-инвариантно. Возможны следующие случаи.

Случай 1: $I_{z} \cap \operatorname{Pr}\left(\mathbf{B}_{1}\right) \neq \varnothing$. В силу транзитивности $P$ на $\operatorname{Pr}\left(\mathbf{B}_{1}\right)$ имеем $\operatorname{Pr}\left(\mathbf{B}_{1}\right) \subseteq I_{z}$. Так как $z \neq 1$, то в силу леммы 5 , п. (1) $I_{z}=\operatorname{Pr}\left(\mathbf{B}_{1}\right)$ и, в частности, $I_{z} \cap \operatorname{Pr}\left(\mathbf{B}_{2}\right)=\varnothing$. Рассматривая действие $z$ на базисе $\operatorname{Pr}\left(\mathbf{B}_{1}\right) \cdot \operatorname{Pr}\left(\mathbf{B}_{2}\right)$, получаем $\chi(z)=0$. Значит, в силу леммы $2 b(z)=s(z)+1-\chi(z)=n+1$, т.е. $z-$ $\mathrm{WP}$-трансляция.

Случай 2: $I_{z} \cap \operatorname{Pr}\left(\mathbf{B}_{2}\right) \neq \varnothing$. Рассматривается аналогично случаю 1.

Случай 3: $I_{z} \cap\left(\operatorname{Pr}\left(\mathbf{B}_{1}\right) \cup \operatorname{Pr}\left(\mathbf{B}_{2}\right)\right)=\varnothing$. Рассматривая действие $z$ на базисе $\operatorname{Pr}\left(\mathbf{B}_{1}\right) \cdot \operatorname{Pr}\left(\mathbf{B}_{2}\right)$, получаем $\chi(z)=0$. Далее имеем

$$
b(z) \geqslant 2 \Rightarrow s(z)=\chi(z)+b(z)-1=b(z)-1 \geqslant 1 .
$$

Пусть неподвижный относительно $z$ примитивньй идемпотент $e \in \operatorname{Pr}(\mathscr{D})$ принадлежит подалгебре $\mathbf{B}_{i}, i \neq 1,2$. Тогда в силу максимальности порядка $P$ является силовской $p$-подгруппой группы $G_{1, i}$. В силу леммы $16 P$ действует транзитивно на $\operatorname{Pr}\left(\mathbf{B}_{i}\right)$. Следовательно, $\operatorname{Pr}\left(\mathbf{B}_{i}\right) \subseteq I_{z}$ и аналогично случаю 1 получаем, что $z$ является WP-трансляцией.

Итак, во всех случаях группа $G=$ Aut $\mathscr{D}$ содержит нетривиальную WP-трансляцию. Из неприводимости $G$ на пространстве матриц с нулевым следом вытекает транзитивность $G$ на $\operatorname{Pr}(\mathscr{D})$. Следовательно, по теореме $1 \mathscr{D}$ сопряжено J-разложению. Теорема доказана.

\section{Список литературы}

1. Wagner A. On finite affine line transitive planes // Math. Z. 1965. V. 87. P. 1-11.

2. Hughes D. R., Piper F. C. Projective planes. Berlin: Springer, 1982.

3. Иванов Д. Н. Ортогональнее разложения полупростых ассоциативных алгебр // Вестн. МГУ. Сер. 1. Матем., мех. 1988. Т. 43. №1. С. 10-15.

4. Кострикин А. И., Кострикин И. А., Уфнаровский В. А. Ортогональные разложения простых алгебр Ли типа $A_{n} / /$ Труды МИАН. 1981. Т. 158. С. 113-129.

5. Иванов Д. Н. Аналог теоремы Вагнера для ортогональных разложений алгебры матриц $\mathrm{M}_{n}(\mathbb{C}) / /$ УМН. 1994. Т. 49. №1. С. 215-216.

6. Кострикин А. И., Фам Хыу Тьеп. Классификация неприводимых разложений простых алгебр Ли типа $A_{n} / /$ Алгебра и анализ. 1992. №3. С. 571-593.

7. Иванов Д. Н. Ортогональные разложения простых алгебр Ли типа $A_{p^{n}-1}$ и $D_{n}$ с конечным числом классов подобных инвариантных подрешеток // Вестн. МГУ. Сер. 1. Матем., мex. 1989. T. 44. № 2. С. 40-43.

8. Kostrikin A. I., Pham Huu Tiep. Orthogonal decompositions and integral lattices. Berlin: Walter de Gruyter, 1994.

9. Bahturin Yu. A., Sehgal S. K., Zaicev M. V. Groups gradings on associative algebras // J. Algebra. 2001. V. 241. P. 677-698. 
10. Иванов Д. Н. Ортогональные разложения ассоциативных алгебр и сбалансированные системы идемпотентов // Матем. сб. 1998. Т. 189. №12. С. 83-102.

11. Иванов Д. Н. Ортогональные разложения простых алгебр Ли типа $A_{p^{n}-1}$ и изотропные расслоения // УМН. 1987. Т. 42. № 4. С. 141-142.

12. Kantor W. M. Spreads, translation planes and Kerdock sets. I // SIAM J. Algebra Disc. Methods. 1982. № 2. P. 151-165.

13. Kantor W. M. Spreads, translation planes and Kerdock sets. II // SIAM J. Algebra Disc. Methods. 1982. № 3. P. 308-318.

14. Kantor W. M. Expanded, sliced and spread spreads // Finite geometries. Proc. Conf. Washington State Univ., Pullman, 1981. New York: Marcel Dekker, Inc., 1983. P. 251-261. (Lecture Notes in Pure and Appl. Math. V. 82.)

15. Холл М. Теория групп. М.: ИЛ, 1962.

16. Gleason A. M. Finite Fano planes // Amer. J. Math. 1956. V. 78. P. 797-807.

17. Супруненко Д. А. Групшы матриц. М.: Наука, 1979.

18. Isaacs I. M. Character theory of finite groups. New York: Academic Press, 1976.

Тверской государственнњй университет

E-mail: dni@tvcom.ru

Поступила в редакцию 28.10 .2003 